\title{
Natural sweeteners
}

Karthik Balachandran

Assistant Professor of Endocrinology, Sri Ramachandra Medical College \& Research Institute, Chennai, India

Abstract The artificial sweeteners, though marketed as zero calorie food additives, have been found to have several health hazards.Ironically they seem to worsen the metabolic syndrome - the very same disease they are supposed to treat.Natural sweeteners are an alternative to the artificial sweeteners and can be particularly helpful in patients who are newly diagnosed with diabetes. They help in transitioning the patient to a healthier life style. This narrative review, discusses the evidence for and against the use of natural sweeteners in the context of diabetes.

Keywords: Diabetes, natural sweeteners, Stevia

Address for correspondence: Dr. Karthik Balachandran, 23, Mothilal Nagar, $2^{\text {nd }}$ Cross, Moolakulam, Puducherry - 605010 , India.

E-mail: karthik2k2@outlook.com

Received: 20.07.2017, Accepted: 21.07.2017

\section{INTRODUCTION}

Diabetic patients can have a hard time getting used to the idea of a lifetime without sweets. This vexing problem can be circumvented by sweeteners. These sweeteners have very low or no calories and are thus attractive options for the diabetic patient who does not want to forgo his sweet pleasures. However, not all sweeteners are created equal. The sweeteners generally fall into two categories:

1. Natural

2. Artificial.

Table 1 shows the various commonly available artificial and natural sweeteners.

It is ironical that artificial sweeteners entered the market much before the natural sweeteners. However, they tended to have several drawbacks.

\begin{tabular}{|l|l|}
\hline \multicolumn{2}{|c|}{ Access this article online } \\
\hline Quick Response Code: & Website: \\
\hline & www.joshd.net \\
\cline { 2 - 2 } & Dol: \\
& Diabetes_20_17 \\
\hline
\end{tabular}

\section{ARTIFICIAL SWEETENERS: THE PROBLEMS}

The artificial sweeteners were substances which produced an intense sweet sensation but did not lead to an increase in blood glucose. They are not absorbed by the gastrointestinal tract (GIT) and are thus theoretically attractive options. However, as their use became more common, several problems surfaced, including association with insulin resistance, weight gain, and dysglycemia. They were also found to be linked to dementia and other illnesses. How can a substance that is not even absorbed by the body cause such wide-ranging metabolic effects? The search led to the uncovering of prebiotic effect. Even though the artificial sweeteners are not absorbed or metabolized by the human GIT, they are extensively metabolized by the gut microbiome. This metabolism leads to accumulation of toxic substances which wipe out the resident bacteria. Eventually, this leads to selection pressure, and there is an increase in Bacteroides and Clostridiales population and a decrease in Lactobacillus population. Thus artificial sweeteners

This is an open access journal, and articles are distributed under the terms of the Creative Commons Attribution-NonCommercial-ShareAlike 4.0 License, which allows others to remix, tweak, and build upon the work non-commercially, as long as appropriate credit is given and the new creations are licensed under the identical terms.

For reprints contact: reprints@medknow.com

How to cite this article: Balachandran K. Natural sweeteners. J Soc Health Diabetes 2018;6:8-10. 
Table 1: Commonly available natural and artificial sweeteners

\begin{tabular}{ll}
\hline Artificial sweetener & Natural sweetener \\
\hline Saccharin & Stevia \\
Acesulfame & Monk fruit (Siraitia grosvenorii) \\
Aspartame & Licorice root \\
Sucralose & Miracle berry (synsepalum dulcificum) \\
HFCS & Kateme fruit \\
Cyclamate & \\
Neohesperidin dihydrochalcone &
\end{tabular}

HFCS: High-fructose corn syrup

can affect the microbiome, and the dysbiosis is associated with metabolic syndrome.

This risk is not similar across different artificial sweeteners. While saccharin is the most important culprit, the safety of other agents is not certain either.

\section{NATURAL SWEETENERS}

The problems associated with artificial sweeteners have made them unattractive to many diabetes patients. Not only are they potentially unsafe, but also many are associated with a bitter after taste, in more ways than one. This leads to the question - are there any natural alternatives? Are the natural alternatives safer than the artificial sweeteners? We will look at the two natural sweeteners.

\section{MONK FRUIT}

Monk fruit (Siraitia grosvenorii) is a subtropical melon grown in Southeast Asian countries. Legend has it that the fruit derives its moniker by virtue of having been cultivated by Chinese monks $>800$ years ago. Since the fruit is hard to store, it is usually not used in the fresh form. It becomes brown on drying. From the dry fruit, its active ingredient is extracted. These are glycosides - mainly mogrosides. (Siamenoside and neomogroside are other glycosides)..$^{[1]}$ Thus, even though the fruit is natural, the glycosides which are responsible for the sweet taste (250 times more than sugar) make up only about $1 \%$ of the fruit, which are extracted by chemical methods. Animal studies show that the active ingredient of the monk fruit extract can reduce oxidative stress. ${ }^{[2]}$

Apart from being a natural sweetener, monk fruit has several other medicinal properties - antitussive, phlegm-relieving, and antiasthmatic effects. ${ }^{[3]}$ Such pleiotropic actions may be related to the presence of other ingredients such as flavonoids, coumarins, and sterols in the fruit.

\section{Safety}

Rigorous safety studies have not been carried out - partly because of the difficulty in standardizing the production of monk fruit extract. The US Food and Drug Administration has given a generally recognized as safe $\mathrm{e}^{[4]}$ notice about its safety.

\section{Disadvantages}

- Availability and cost - monk fruit is not commonly available. Because of difficulty in growing and the expense of importing, it is generally costlier

- Purity - since it is extremely sweet in tiny quantities, it is often mixed with other substances including dextrose, to add bulk. This can result in variable purity of different preparations

- Lack of rigorous studies shows long-term efficacy.

\section{STEVIA}

Stevia rebaudiana is a plant belonging to the sunflower family (Asteraceae). It is native to Paraguay and Brazil and is over 200 times sweeter than sucrose. It owes this property to the presence of glycosides - stevioside and rebaudioside. Unlike monk fruit, it is much easier to cultivate and the leaves can be eaten fresh. The ease of cultivation in small urban households and the cost, along with its status as a nonnutritive sweetener, makes it an attractive choice.

Initial concerns over carcinogenicity were found to be baseless, and Stevia has been approved for human consumption in both the United States and Europe. Indeed Stevia has been approved for use in a variety of food products in India by FSSAI.

Most Stevia products commercially available are indeed a purified form of rebaudioside $\mathrm{A}$, a component of the Stevia plant. Because of its better availability, Stevia is also better studied in the literature.

\section{EFFICACY}

In a study by Anton et al., ${ }^{[5]} 19$ lean and 12 obese participants were given preloads of Stevia-, aspartame-, and sucrose-laced foods. Stevia significantly reduced postprandial glucose and insulin levels, when compared to other sweeteners. However, not all studies favor the use of intense sweeteners.

Tey et al. studied the effect of Stevia, monk fruit, aspartame, and sucrose on postprandial glucose, insulin, and energy intake. Thirty healthy males took part in this randomized crossover study. It was found that the calorie deficit achieved by taking sweeteners in one meal is compensated for by increased intake in the next meal. Thus, intake of Stevia or other sweeteners did not have a significant effect on postprandial glucose or insulin. However, this study lasted only $24 \mathrm{~h}$. Since the 
benefits (or harms if any) of sweeteners are effected through metagenomic regulation, the effects are difficult to demonstrate in such a short time. In another study by the same author, $24 \mathrm{~h}$ glucose profile was compared between nonnutritive sweeteners and sucrose. No significant difference was found. ${ }^{[6]}$

In a systematic review of published literature on intense sweeteners (which included both natural and artificial sweeteners), it was found that the overall effect on long-term health is inconclusive. Considering the cost of these agents, it might be difficult to justify their use in diabetics. However, this review did not specifically look at Stevia or any other natural agent. ${ }^{[7]}$ Rogers et al. ${ }^{\left[{ }^{[8]}\right.}$ in a systematic review and meta-analysis, looked at both human and animal studies. In sustained intervention randomized controlled trials (from 4 to 40 weeks), there was a significant reduction in body weight in those who took low-energy sweeteners. Thus, the weight of evidence favors the use of low-energy sweeteners such as Stevia over a longer period.

\section{Disadvantages}

- Expense - purified Stevia products are more expensive than sugar. However, because of the ease of growing Stevia in small pots in homes and the fact that fresh leaves/its extracts can be used, it is much better suited to Indian diabetics than other sweeteners

- Purity - Like any other "natural" product, commercially available preparations can have different purity and the addition of sucrose/dextrose to Stevia may nullify its advantages

- Availability

- Relative paucity of long-term data

- Allergic reaction - allergy to other members of the Asteraceae family such as daisies, ragweed, chrysanthemum, and sunflower can cause allergy to Stevia.

\section{CONCLUSION}

The ever-increasing burden of diabetes and the disenchantment with artificial sweeteners has created a public demand for natural sweeteners. While the weight of medium-term data supports the use of natural sweeteners, especially Stevia, we should be cautious about recommending natural sweeteners to vulnerable population - children and pregnant women. The methodological flaws in published studies make it difficult to come to firm conclusions regarding the superiority of nonnutritive sweeteners over cessation of sugar intake.

However, occasional use of such natural sweeteners is unlikely to result in long term. Thus, natural sweeteners may have a place in transitioning newly diagnosed diabetics to a more healthy lifestyle.

Financial support and sponsorship

Nil.

\section{Conflicts of interest}

There are no conflicts of interest.

\section{REFERENCES}

1. Dharmananda S, Dorr C. Luo Han Guo: Sweet Fruit Used as Sugar Substitute and Medicinal Herb; 25 October, 2011. Available from: http://www.itmonline.org/arts/luohanguo.htm. [Last accessed on 2017 Jun 06].

2. Xu Q, Chen SY, Deng LD, Feng LP, Huang LZ, Yu RR. Antioxidant effect of mogrosides against oxidative stress induced by palmitic acid in mouse insulinoma NIT-1 cells. Braz J Med Biol Res 2013;46:949-55.

3. Li C, Lin LM, Sui F, Wang ZM, Huo HR, Dai L, et al. Chemistry and pharmacology of Siraitia grosvenorii: A review. Chin J Nat Med 2014;12:89-102.

4. Center for Food Safety and Applied Nutrition. Generally Recognized as Safe (GRAS): Center for Food Safety and Applied Nutrition. Available from: https://www.fda.gov/food/ingredientspackaginglabeling/ gras/. [Last accessed on 2017 Jun 06].

5. Anton SD, Martin CK, Han H, Coulon S, Cefalu WT, Geiselman P, et al. Effects of stevia, aspartame, and sucrose on food intake, satiety, and postprandial glucose and insulin levels. Appetite 2010;55:37-43.

6. Tey SL, Salleh NB, Henry CJ, Forde CG. Effects of non-nutritive (artificial vs. natural) sweeteners on 24-h glucose profiles. Eur J Clin Nutr 2017;71:1129-32.

7. Olivier B, Serge AH, Catherine A, Jacques B, Murielle B, Marie-Chantal CL, et al. Review of the nutritional benefits and risks related to intense sweeteners. Arch Public Health 2015;73:41.

8. Rogers PJ, Hogenkamp PS, de Graaf C, Higgs S, Lluch A, Ness AR, et al. Does low-energy sweetener consumption affect energy intake and body weight? A systematic review, including meta-analyses, of the evidence from human and animal studies. Int J Obes (Lond) 2016;40:381-94. 